ACCEPTED MANUSCRIPT

\title{
Theory-assisted determination of nano-rippling and impurities in atomic resolution images of angle-mismatched bilayer graphene
}

To cite this article before publication: Oleg Ovchinnikov et al 2018 2D Mater. in press https://doi.org/10.1088/2053-1583/aadb5f

\section{Manuscript version: Accepted Manuscript}

Accepted Manuscript is "the version of the article accepted for publication including all changes made as a result of the peer review process, and which may also include the addition to the article by IOP Publishing of a header, an article ID, a cover sheet and/or an 'Accepted

Manuscript' watermark, but excluding any other editing, typesetting or other changes made by IOP Publishing and/or its licensors"

This Accepted Manuscript is @ 2018 IOP Publishing Ltd.

During the embargo period (the 12 month period from the publication of the Version of Record of this article), the Accepted Manuscript is fully protected by copyright and cannot be reused or reposted elsewhere.

As the Version of Record of this article is going to be / has been published on a subscription basis, this Accepted Manuscript is available for reuse under a CC BY-NC-ND 3.0 licence after the 12 month embargo period.

After the embargo period, everyone is permitted to use copy and redistribute this article for non-commercial purposes only, provided that they adhere to all the terms of the licence https://creativecommons.org/licences/by-nc-nd/3.0

Although reasonable endeavours have been taken to obtain all necessary permissions from third parties to include their copyrighted content within this article, their full citation and copyright line may not be present in this Accepted Manuscript version. Before using any content from this article, please refer to the Version of Record on IOPscience once published for full citation and copyright details, as permissions will likely be required. All third party content is fully copyright protected, unless specifically stated otherwise in the figure caption in the Version of Record.

View the article online for updates and enhancements. 
Theory-assisted determination of nano-rippling and impurities in atomic resolution images of anglemismatched bilayer graphene

\section{Authors}

Oleg S. Ovchinnikov ${ }^{1,2}$, Andrew O'Hara ${ }^{1}$, Ryan Nicholl ${ }^{1}$, Jordan A. Hachtel ${ }^{1,3,4}$, Kirill Bolotin ${ }^{1,5}$, Andrew Lupini $^{4}$, Stephen Jesse ${ }^{2,3}$, Arthur P. Baddorf ${ }^{2,3}$, Sergei V. Kalinin ${ }^{2,3}$, Albina Y. Borisevich ${ }^{2,4}$, Søkrates T. Pantelides ${ }^{1,4,6}$

Affiliations

1: Department of Physics and Astronomy, Vanderbilt University, Nashville, TN, USA

2: Institute for Functional Imaging of Materials, Oak Ridge National Laboratory, Oak Ridge, TN, USA

3: Center for Nanophase Materials Sciences, Oak Ridge National Laboratory, Oak Ridge, TN, USA

4: Materials Sciences and Technology Division, Oak Ridge National Laboratory, Oak Ridge, TN, USA

5: Freie Universität Berlin, Fachbereich Physik, Institut für Experimental Physik, Arnimallee 14, 14195 Berlin, Germany

6: Department of Electrical Engineering and Computer Science, Vanderbilt University, Nashville, TN 37235, USA

oleg.ovchinnikov@vanderbilt.edu, andrew.ohara@vanderbilt.edu 
Notice: This manuscript has been authored by UT-Battelle, LLC, under Contract No. DE-AC0500OR22725 with the U.S. Department of Energy. The United States Government retains and the publisher, by accepting the article for publication, acknowledges that the United States Government retains a non-exclusive, paidup, irrevocable, world-wide license to publish or reproduce the published form of this manuscript, or allow others to do so, for the United States Government purposes. The Department of Energy will provide public access to these results of federally sponsored research in accordance with the DOE Public Access Plan (http://energy.gov/downloads/doe-public-access-plan).

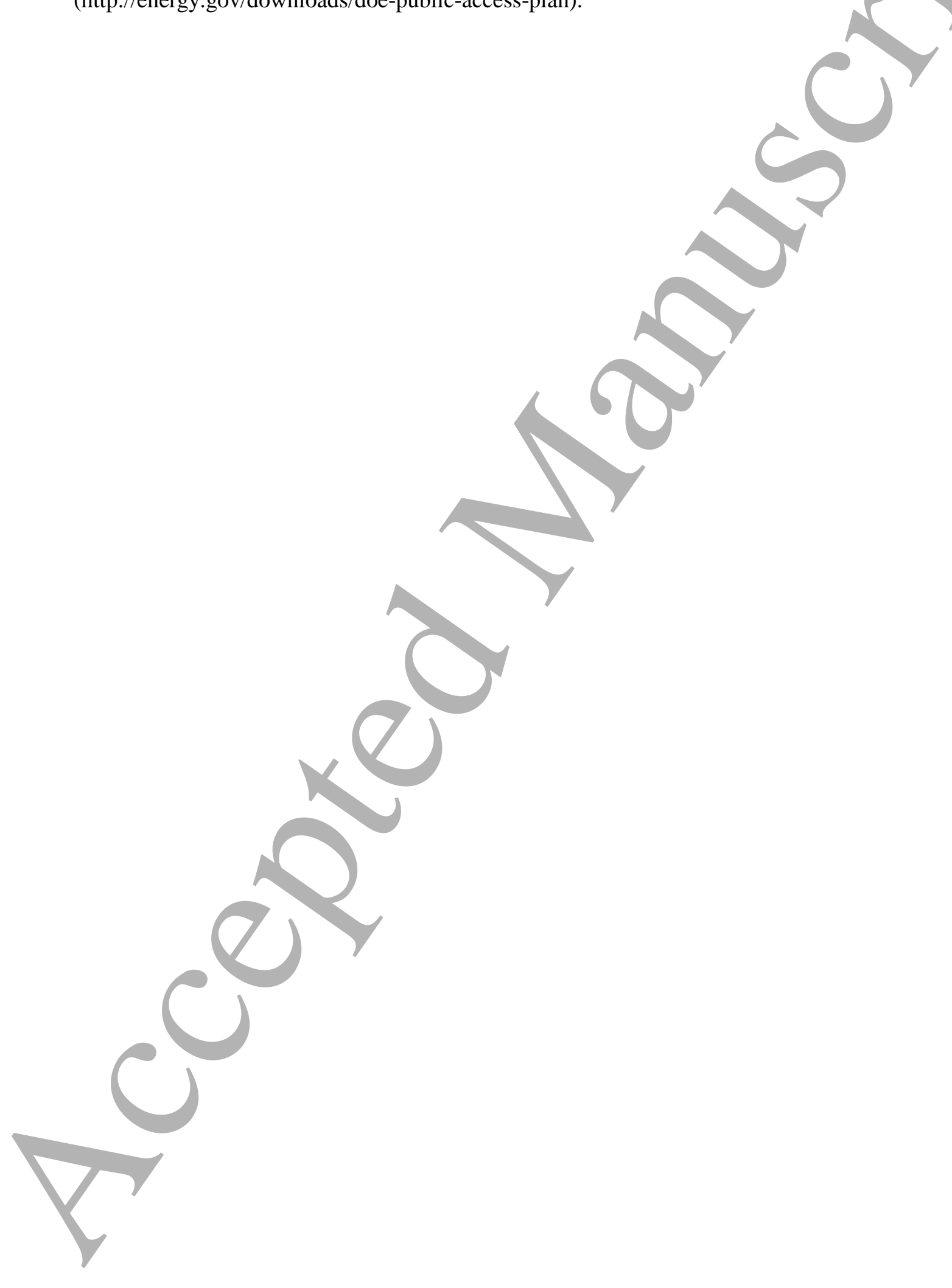


Abstract

Ripples and impurity atoms are universally present in 2D materials, limiting carrier mobility, creating pseudo-magnetic fields, or affecting the electronic and magnetic properties. Scanning Transmission Electron Microscopy (STEM) generally provides picometer-level precision in the determination of the location of atoms or atomic "columns" in the in-image plane ( $x y$ plane). However, precise atomic positions in the $z$-direction as well as the presence of certain impurities are difficult to detect. Furthermore, images containing moiré patterns such as those in angle-mismatched bilayer graphene compound the problem by limiting the determination of atomic positions in the $x y$ plane. Here, we introduce a reconstructive approach for the analysis of STEM images of twisted bilayers that combines the accessible $x y$ coordinates of atomic positions in a STEM image with density-functional-theory calculations. The approach allows us to determine all three coordinates of all atomic positions in the bilayer and establishes the presence and identity of impurities. The deduced strain-induced rippling in a twisted bilayer graphene sample is consistent with the continuum model of elasticity. We also find that the moiré pattern induces undulations in the $z$ direction that are approximately an order of magnitude smaller than the strain-induced rippling. A single substitutional impurity, identified as nitrogen, is detected. The present reconstructive approach can, therefore, distinguish between moiré and strain-induced effects and allows for the full reconstruction of 3D positions and atomic identities.

Keywords

Scanning Transmission Electron Microscope, bilayer graphene, Density Functional Theory, rippling, strain, graphene
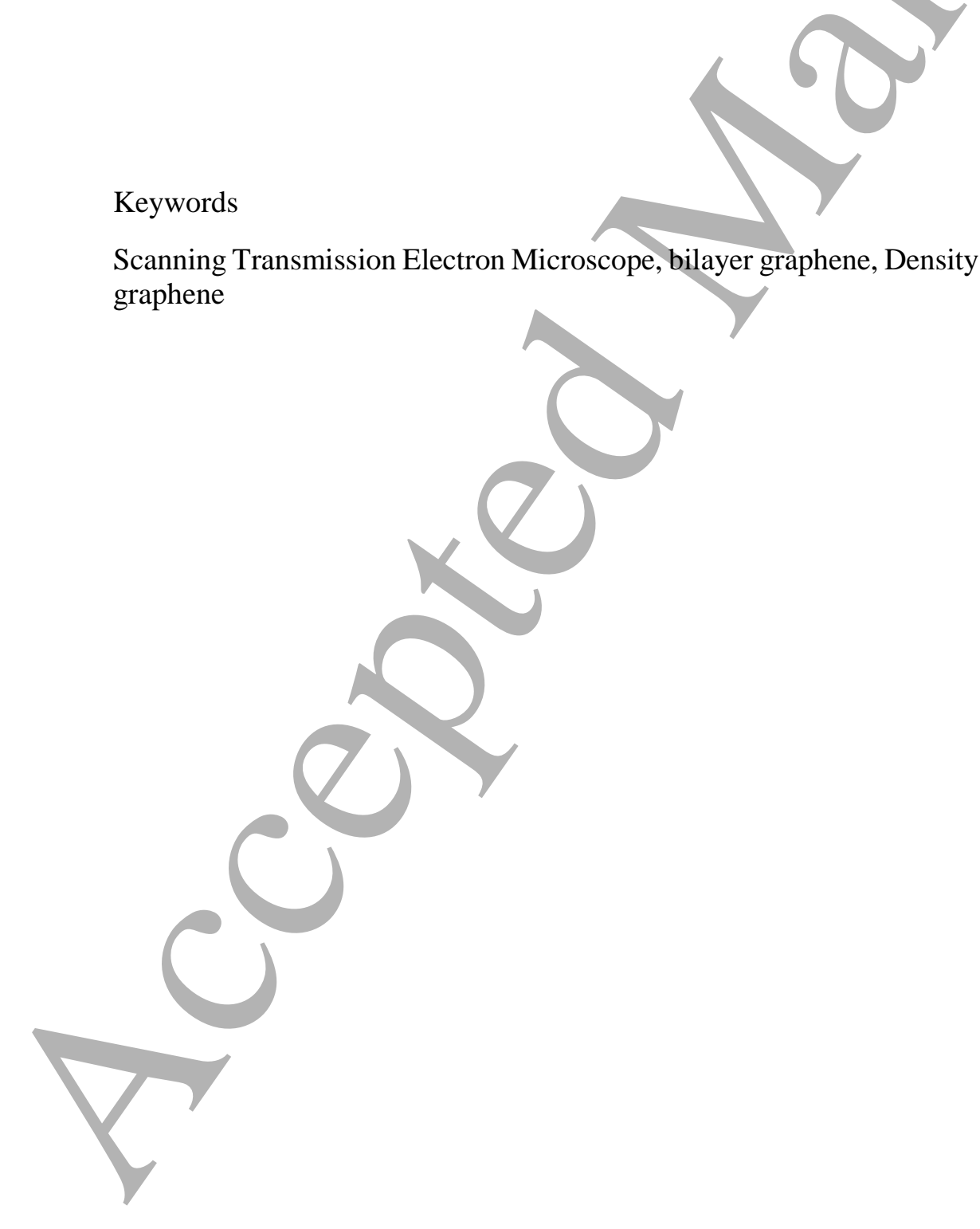


\section{Introduction}

Graphene has been hailed and studied for its wide range of potential electronic and mechanical applications. Rippling has been seen at a variety of length scales in graphene systems [1-6], changes their electronic, magnetic, and other properties [1] and can induce a pseudo-magnetic field [2]. Bilayer graphene has also been extensively studied for a multitude of applications such as diodes, transistors, optoelectronics, and superconductivity [7-14]. For some of these applications, the tunability of its band gap is a valuable feature. Angle-mismatch between the layers of bilayer materials is common and leads to the formation of a moiré pattern. Moiré patterns in two-dimensional (2D) materials often obscure vital information when imaged, such as the underlying layer in Scanning Tunneling Microscopy (STM) and the localization of individual atomic "columns" in Scanning Transmission Electron Microscopy (STEM). Yet, obscured information can play a major role in determining the properties of the material, especially the presence of defects, interstitial dopants between the layers, and rippling, which affect both electronic and magnetic properties [1,2].

Aberration-corrected STEM is a quantitative tool that is capable of locating atomic columns in crystals with picometer-level precision. The ability to achieve sub-pixel precision in the location of the center of an atom or atomic column in STEM images has been demonstrated through the use of center-of-mass and 2D function fitting, which in turn has been used to help determine the properties of complex structures such as thin films, superlattices, and nanoparticles [15-19]. While STEM possesses picometer-scale resolution in the in-image-plane ( $x y$ plane), the resolution perpendicular to the image-plane ( $z$ direction) is approximately a nanometer, which limits the instrument's ability to study rippling in 2D materials. In many important cases, precise determination of the $x y$ coordinates of all atoms or atomic "columns" is not possible, e.g., in bilayers in which an angle mismatch between the layers exists and leads to moiré patterns. In such cases, due to apparent overlaps of atoms in the projected image, it is impossible to determine where one atom ends and another begins. Nevertheless, even in these materials systems, isolated areas exist that have clear and discernible atomic columns where it is possible to locate a subset of atomic coordinates with high precision.

In this paper, we focus on nanoscale rippling in angle-mismatched bilayer graphene (the two layers are rotated with respect to each other) and demonstrate a reconstructive approach that allows us to determine the $x y z$ coordinates of all atoms by using the $x y$ coordinates of only the limited number of discernible atoms in the bilayer image and performing suitable density-functional-theory (DFT) calculations. By combining the information obtained from the distances between moiré nodes (from here on referred to simply as nodes) and those atomic positions that can be accurately extracted from the STEM image, a defect-free reference patch is constructed. DFT is then used to optimize the atomic coordinates in the reference patch under the constraint of the known $x y$ atomic coordinates. By simulating the STEM image using the optimized reference patch, a local-area correlation is done with the experimental image. Areas of low correlation are then attributed to the presence of defects. The atom(s) in areas of low correlation can then be replaced by likely defect candidates and the local-area correlation is repeated until the defect causing the low correlation is identified. In the present case, only a single atomic site exhibits low correlation and we are able to identify it as substitutional nitrogen. The $z$ information for each layer can further be used to quantify the rippling in the sample. In the case at hand, the predominant source of rippling is determined to be caused by strain in the sample. Furthermore, the distance between the two layers can be used to determine the moiré-patterninduced undulations, which are in agreement with those previously predicted by theory [20]. The reconstructive analysis of bilayers, which is accomplished by combining STEM with DFT to determine atomic coordinates in three dimensions, while potentially computationally expensive, allows for a complete characterization of nano-rippling in a way that is impossible from imaging alone.

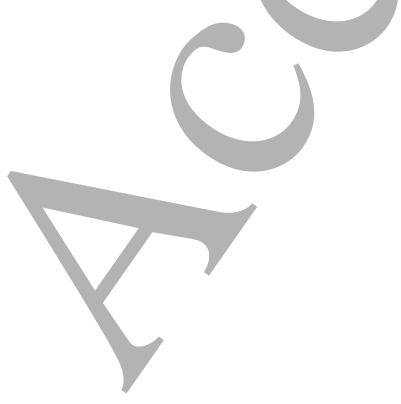




\section{Methods and Materials}

\subsection{Sample preparation and data collection}

High-quality graphene was grown on copper foils using atmospheric-pressure chemical vapor deposition (CVD) [21]. The graphene was subsequently transferred to TEM grids [22] following a direct transfer procedure [13]. A TEM grid was placed upside down directly on top of a copper foil coated with CVD graphene forming a stack. A drop of isopropyl alcohol (IPA) was applied to the stack. Upon evaporation of the IPA, graphene was pulled from the copper surface and adhered strongly to the TEM grid. Excess copper was removed with CE-100 copper etchant. The remaining TEM grid with graphene was rinsed in three baths of deionized water and IPA. This procedure resulted in large coverage of residue-free monolayer graphene and, critically, some regions of bilayer graphene originating from monolayer graphene that folded during transfer.

The bilayer graphene was experimentally analyzed using annular dark field (ADF) imaging on a Nion aberration-corrected UltraSTEM 100, operated at an accelerating voltage of $60 \mathrm{kV}$ [23]. Figure 1(a) shows an atomic-resolution ADF image (1024 x 1024 pixels) of angle-mismatched bilayer graphene. To reduce noise in the image, Figure 1(a) was filtered with a Gaussian blur [24] with a standard deviation of two pixels, producing figure 1(b). Figure 1(b) shows both the atomic columns of individual carbon atoms and the moiré pattern caused by the incommensurate rotation angle.

\subsection{Determining the angle of relative rotation}

To determine the angle of relative rotation between the two layers of graphene (figure 1a-1b), a Gaussian blur with a sigma of 10 pixels was applied to the original image (figure 1c-d). From this image, it is clear

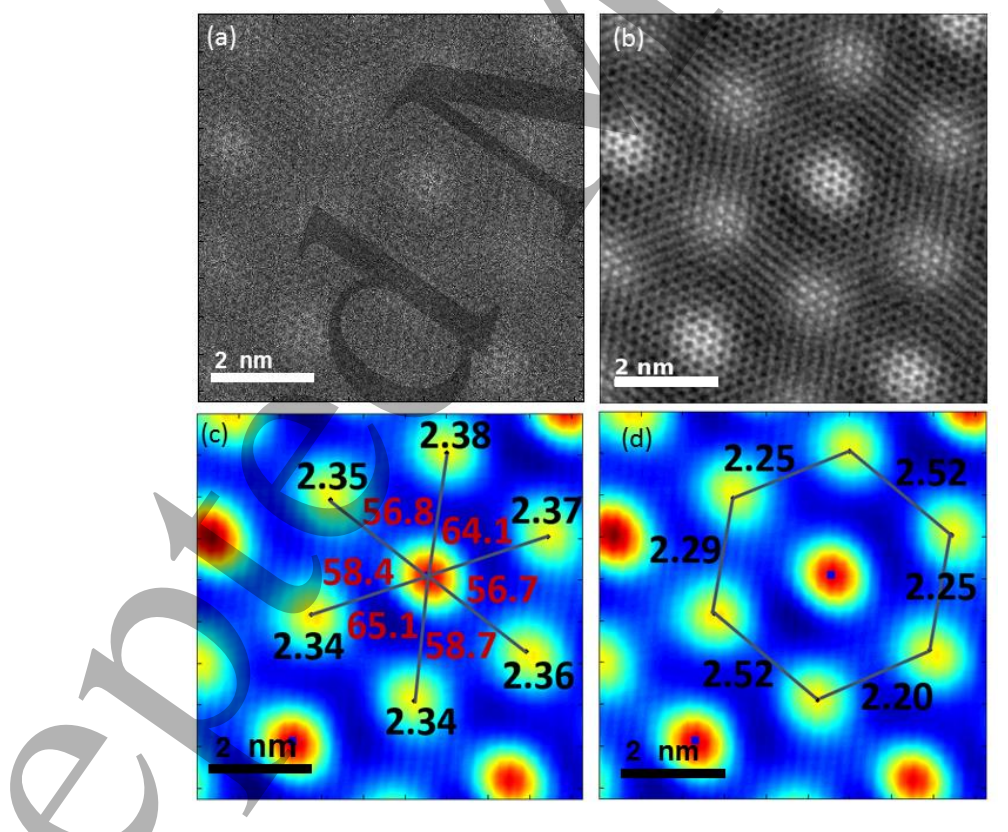

Figure 1. (a) ADF STEM image of a graphene bilayer with relative rotation between the layers (mismatched or twisted graphene bilayer), as acquired. (b) The same image with a small Gaussian blur more clearly shows the presence of two types of moiré nodes in the moiré pattern (primary higher intensity, secondary - lower intensity). (c-d) The same image with a heavy Gaussian blur used to indicate the deviations from a perfect moiré pattern due to strain. (c) Distances between secondary and primary nodes (in nm: black) and angle between secondary nodes around a central primary node (in degrees: red). (d) Distances between secondary nodes (in nm: black). 
that there are two different types of moiré nodes in the moiré pattern: brighter nodes that correspond to areas of highest lattice correlation (primary nodes, AA stacking) and dimmer nodes that correspond to areas of highest anti-correlation (secondary nodes, AB stacking) of the two lattices. In order to determine the angle of relative rotation between the graphene layers, the distance between the moire nodes must be determined. The moiré nodes were isolated by first using an image threshold of $75 \%$ of the maximum intensity followed by density-based clustering [25]. Once the moiré nodes where isolated, a center-of-mass algorithm was used to find the center of each node while the average intensity of the node was used to determine if a node is primary or secondary. Using the center point of each moiré node, the distance between a primary node and its surrounding secondary nodes (Figure 1c), the distance between the secondary nodes around the primary node (Figure 1d), and the angle separating the secondary nodes around the primary node (Figure 1c) were calculated. These distances and angles are not uniform, implying the presence of strain in the sample.

The angle $\theta$ of relative rotation between the two layers can then be determined from the primary-to-primary moiré-node distance via [26]:

$$
L(\theta)=\frac{a b}{\sqrt{a^{2}+b^{2}-2 a b \cos \theta}}
$$

where $\mathrm{a}$ and $\mathrm{b}$ represent the lattice constants of the two layers. For bilayer graphene, both are $2.46 \AA$. By substituting these values into Eq. 1, we obtain:

$$
L(\theta)=\frac{2.46}{2 \sin \theta / 2}
$$

Due to the variance in the moiré node distances, an average node-to-node distance was used to find the angle of relative rotation. To improve the average, not only were the primary-to-primary moiré node distances $\left(L_{p p}\right)$ used, but secondary-to-primary moiré-node distances $\left(L_{p s}\right)$ where converted into related primary-to-primary moiré-node distances using simple trigonometry:

$$
L_{p p}=2 \cos \left(30^{\circ}\right) L_{p s}=\sqrt{3} L_{p s} .
$$

Using the average node-to-node distance, the angle of relative rotation was found to be $4.45^{\circ}$ in the present case.

\subsection{Filtering image and identification of individual atomic columns}

In order to identify individual atomic positions, the original image was filtered to remove unwanted image collection phenomena such as noise and surface contamination. Due to the nature of the moiré interference, atomic columns can only be distinguished around the center of a moiré node, whereby it is only necessary to filter these areas. Using the center of each moiré node, a box 201x201 pixels was removed from the image for filtering. A similar box area extraction (Figure 2) was used throughout this paper and is referred to as "local area extraction". 


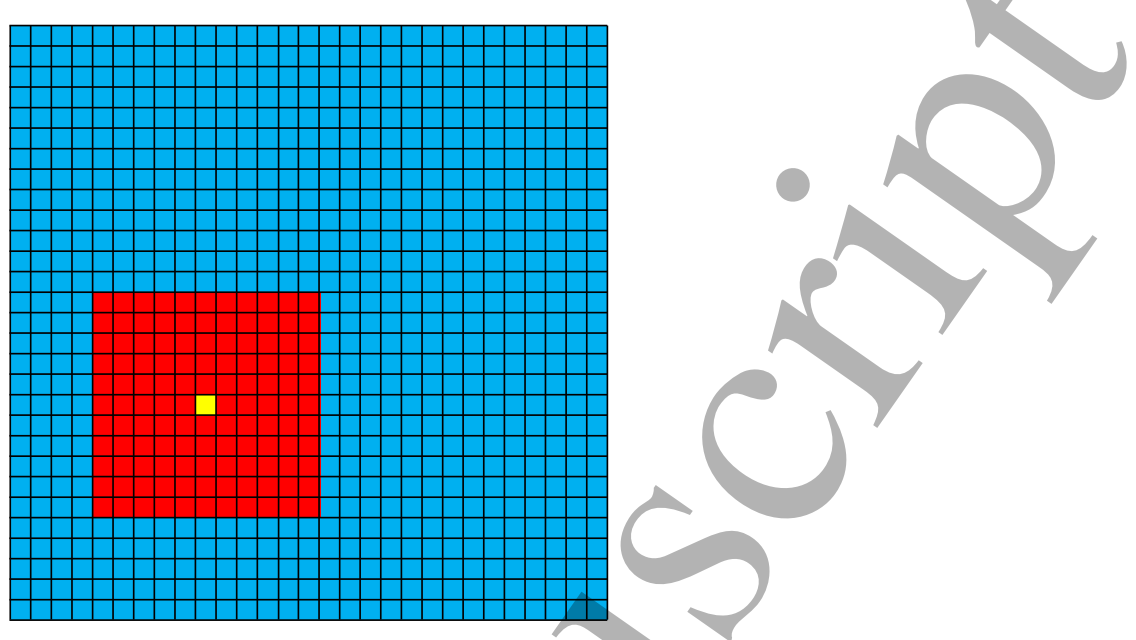

Figure 2. Example of local area extraction of a 11x11 area (red and yellow areas) around a center pixel (yellow area) from a 29x29 pixel image.

The extracted areas around moiré nodes were filtered using a previously reported filtering technique that utilizes principal component analysis (PCA) to remove noise and surface contamination from the image[27]. This filtering technique requires slicing the image into smaller sub-images, each of which is then filtered separately using statistical correlations to remove noise and surface contamination. The full image is then reconstructed from these filtered sub-images.

For the secondary moiré nodes, this level of filtering is adequate. However, for primary moiré nodes where distinguishable atomic columns are closer together, an additional filtering step was necessary. This filtering step consists of a 2D correlation with an ideal atomic column (a 2D Gaussian). At each pixel, a local area (Figure 2) of 21x21 pixels was extracted. These areas where passed through a 2D cross-correlation [28] defined by

$$
r(A, B)=\frac{\sum_{m} \sum_{n}\left(A_{m n}-\bar{A}\right)\left(B_{m n}-\bar{B}\right)}{\sqrt{\left(\sum_{m} \sum_{n}\left(A_{m n}-\bar{A}\right)^{2}\right)\left(\sum_{m} \sum_{n}\left(B_{m n}-B\right)^{2}\right)}},
$$

with A being the extracted area and B a 2D Gaussian with a sigma of 13 pixels. In each node, the atomic columns were identified using two common image analysis techniques, intensity thresholding followed by density-based clustering [25].

\subsection{Creating the initial unit cell and DFT calculations}

DFT simulations of the entire image is computationally prohibitive due to the number of atoms required to make a periodic image with a small relative rotation angle. Instead, a patch was used. We considered an area of the image that encompasses two primary and two secondary nodes and constructed a corresponding idealized patch by taking two pristine graphene lattices overlaid in an AA-stacking configuration and a separation of $3.5 \AA$. The top lattice was then rotated to the desired angle of relative rotation $\left(4.45^{\circ}\right)$ using the center of a hexagon as the rotation point. The atomic coordinates in this idealized bilayer graphene patch are then replaced by the coordinates of the atomic columns that where determined from the experimental image. Protruding atoms that contain only a single carbon-carbon bond were removed from the edge of the patch. Edge atoms with fewer than three neighbors were passivated with hydrogen atoms. 


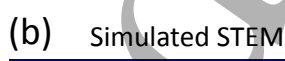

(a) Relaxed positions

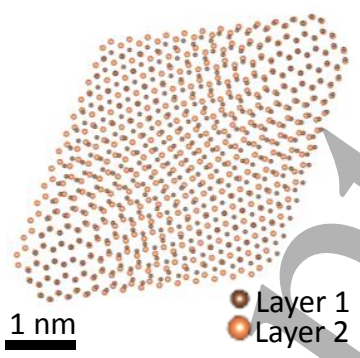

The constructed bilayer patch consists of 899 carbon atoms and 111 hydrogen atoms, with the patch spanning $45.64 \times 39.31 \times 3.5 \AA$. The patch was placed into a unit cell spanning $55 \times 55 \times 25 \AA$. DFT calculations were performed using the Vienna ab initio Simulation Package (VASP) [29] with the Brillouin zone sampled at the $\Gamma$ point only. The exchange-correlation functional was described via the Perdew-BurkeErnzerhof parametrization [30] of the generalized gradient approximation. A van der Waals dispersion correction was included via Grimme's DFT-D3 method [31] and the DFT-D3 plus Becke-Jonson damping method [32]. The DFT-D3(BJ) correction produced results in better agreement with the experimental data as in a previous study of bilayer graphene by Lebedeva et al. [33]. The projector-augmented wave (PAW) method $[34,35]$ was used to describe the interaction between core and valence electrons and the plane-wave basis-set cutoff energy was set as $400 \mathrm{eV}$. During structural optimization, the $x$ - and $y$-coordinates of atoms that could be determined from the experimental data were held fixed while the $x$-and $y$-coordinates of the rest of the atoms and the $z$-coordinates of all atoms were allowed to move until all residual forces were less than $0.01 \mathrm{eV} / \mathrm{A}$.

\section{Results and Discussion}

To test how well theory recovered the atomic coordinates of all the atoms, the patch described above was removed from the original image and filtered in its entirety using the PCA filtering as in Section 2.3. The DFT-optimized patch (Figure 3a) was used to simulate the STEM image using the QSTEM simulation software [36] (Figure 3b). The beam parameters for the simulation were taken from those used during the experimental image acquisition, with the focus varied until the simulation was visually similar to that in the filtered patch (Figure 3c). To compare the simulated and experimental images, a local-area 2D crosscorrelation (Eq. 4) was employed between the filtered and simulated STEM images. This procedure necessitated the interpolation of the simulated image on to the same coordinate system as the experimental image. Once the two images were in the same coordinate system, a local area (Figure 2) of $27 \times 27$ pixels was extracted from both images at each pixel. The correlation between simulation and experiment is very good, over 0.8 in most places, with 1.0 indicating perfect correlation (Figure 3d), providing confidence that the deduced atomic positions are correct or extremely close to the actual positions. Any defects in the experimental lattice should appear as areas of low correlation.

Figure 3. (a) Atomistic model showing the optimized positions of all carbon atoms with the two layers in different colors (b) PCA filtered simulated image of the post-DFT-optimized locations. (c) PCA filtered patch from the original image. (d) Map of local $2 \mathrm{~d}$ correlation coefficients between the filtered simulated image and the filtered experimental image.
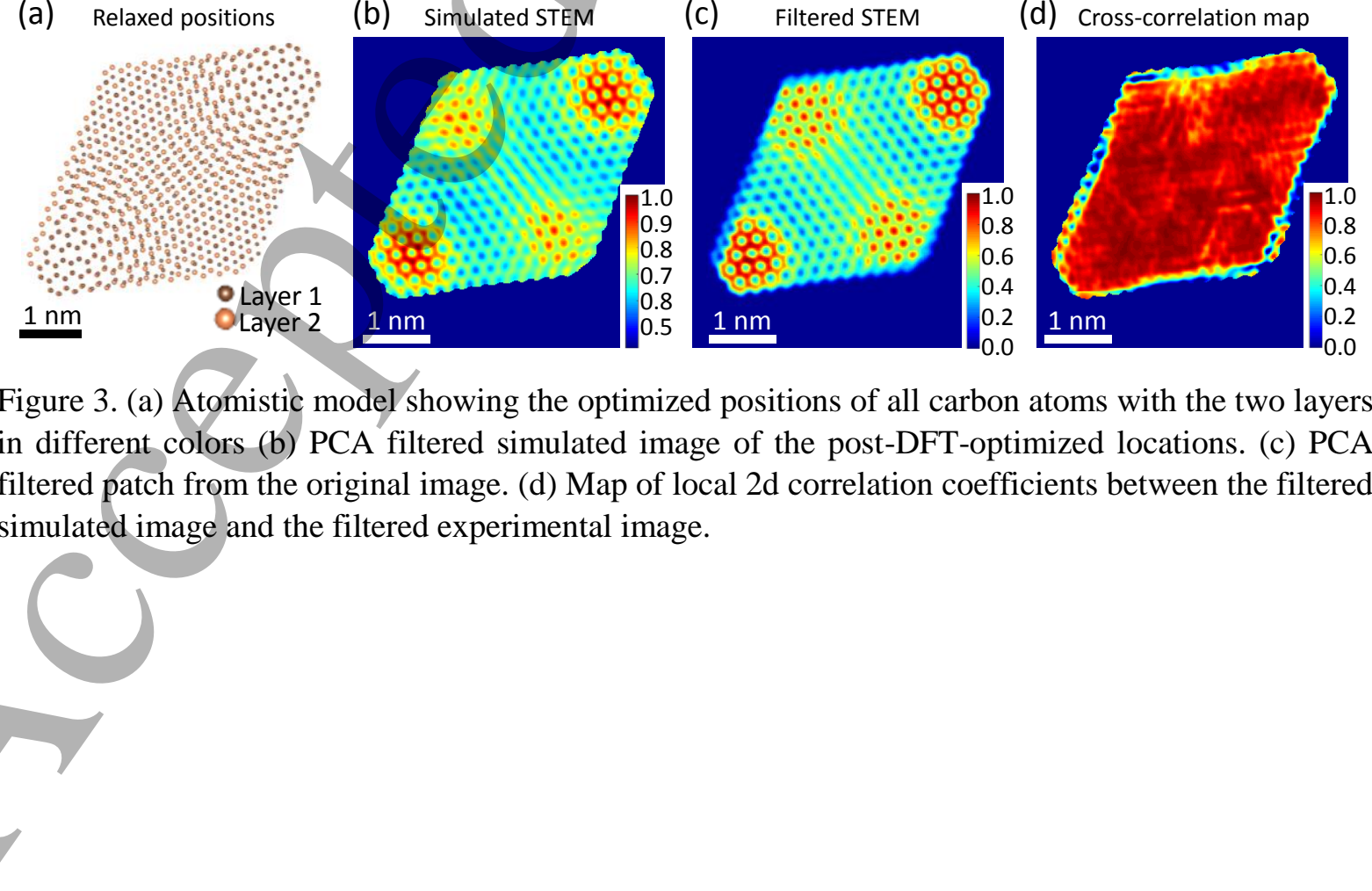
(a) Overall cross-correlation

(b) Layer 1 cross-correlation

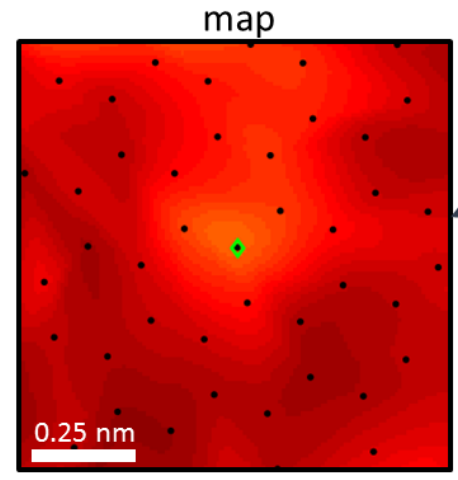

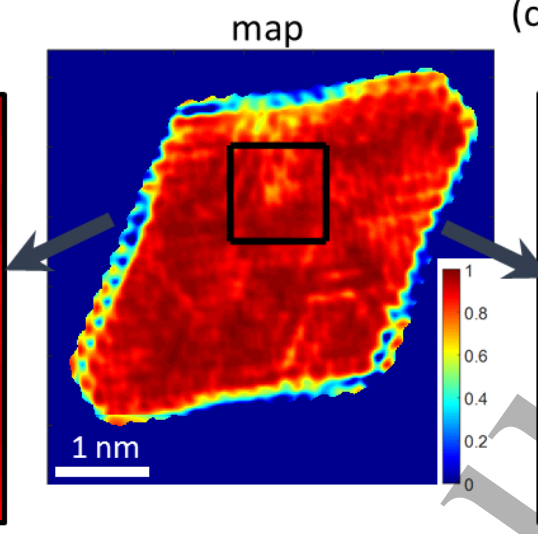

(c) Layer 2 cross-correlation

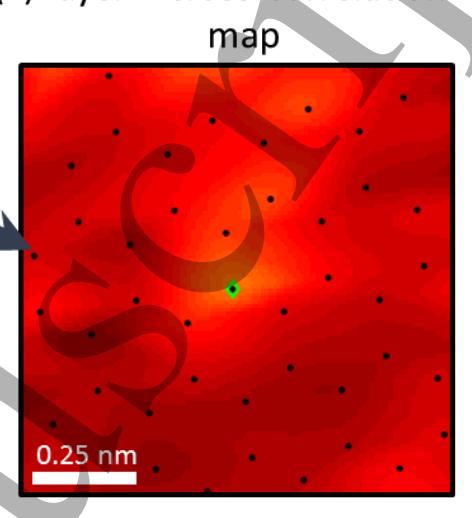

Figure 4. (a) 2D cross-correlation map with area of interest highlighted and separated into (b) layer 1 with atom of interest highlighted and (c) layer 2 with atom of interest highlighted.

While edge effects cause areas of low correlation to occur near the edge of the patch, one significant area of low correlation just above the center of the patch stands out (figure 4a). In order to determine if this area of low correlation is caused by a defect or is a limitation of the present reconstructive-analysis method, the most probable defects can be inserted into the optimized DFT patch and the patch re-optimized. First, however, the coordinates of the atom(s) that cause the low correlation must be determined by separating the 2D cross-correlation map into a cross-correlation map for each layer (Figure 4b-4c). This separation is done by extracting local areas (Figure 2) from the 2D cross-correlation image at every atom in the lattice using a box $21 \times 21$ pixels. These local areas are then averaged and the results are interpolated onto the coordinate system of the $2 \mathrm{D}$ cross-correlation image. We find that the observed area of low correlation is centered in each layer on a single atom. The $x y$ coordinates of these atoms are extremely close together, making it impossible to determine which layer contains the defect. Although it is impossible to determine which layer the potential defect is in, it is possible to narrow it to a single atom in each layer (Figure 4b$4 c)$

With the possible location of the defect causing the low correlation narrowed down to two potential atomic sites determination of the defect type was performed. A set of the most likely defects was analyzed including a carbon monovacancy and substitional boron, nitrogen, and oxygen atoms. The previously optimized DFT patch was modified and re-optimized, with each defect type tested at each of the two candidate sites. A simulated STEM image was then generated for each patch and new 2D cross-correlation maps were calculated to determine if any of these defects improved the overall correlation (Figure 5a). These new 2D cross-correlation maps were compared with the original defect-free scenario visually and statistically using the percent change in cross-correlation around the area of low correlation (Figure 5). Based on both the visual and statistical comparisons, the low correlation is most likely caused by a substitional nitrogen defect in layer 2 .

The $z$ information from the optimized DFT positions was interpolated onto a square lattice. The optimized $z$ displacements show two clear ripples in both layers (Figure 6) in the primary-primary node direction. These ripples have a height of $\sim 0.75 \AA$ (using the distance of highest to lowest atom in each layer's graphene lattice) and the peak-to-peak distance of the two ripples is $\sim 1.5 \mathrm{~nm}$. When comparing the rippling predicted by the optimized reference patch with the experimental image, a correlation between the rippling and the background intensity can be visually observed. To determine the exact wavelength and amplitude of the 
(a) Change in 2D cross-correlation

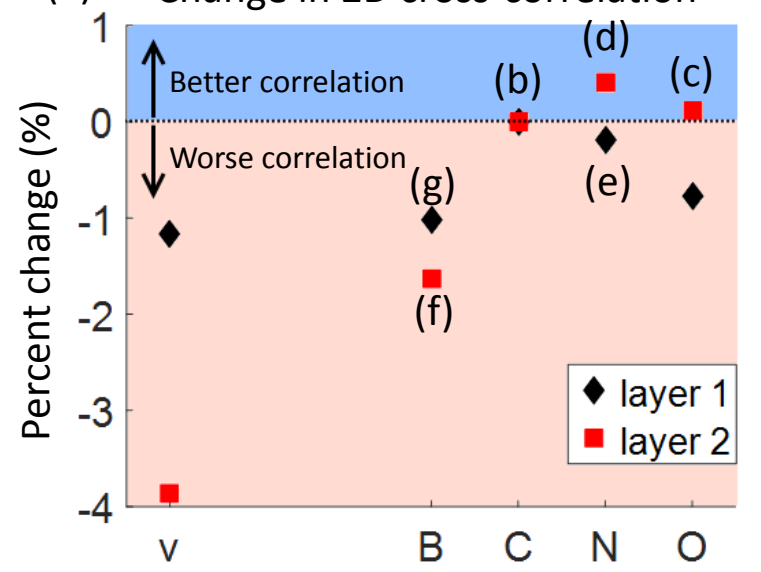

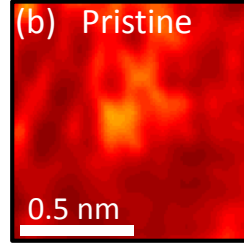
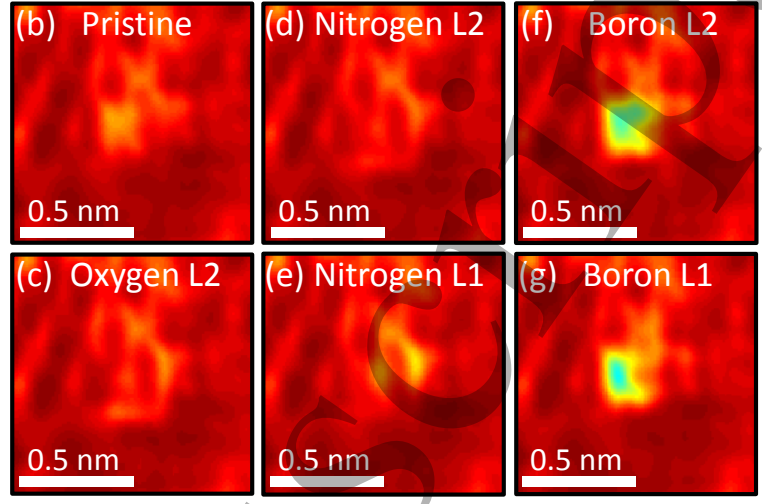

Figure 5. (a) Plot of the effect on the local 2D cross correlation when atoms are substituted, (b) zoomed in area of cross-correlation map for pristine bi layer graphene, (c) Carbon in layer 2 is replaced by oxygen, (d) Carbon in layer 2 is replaced by nitrogen (e) Carbon in layer 1 is replaced by nitrogen, (f) Carbon in layer 2 is replace by boron, and ( $\mathrm{g}$ ) Carbon in layer 1 is replaced by boron.

rippling, the positions of the atoms were treated as a grid of points with coordinates $(x, y, z)$. The atomic positions were then interpolated onto a square grid with a tight spacing between points, $\sim 0.1 \AA$ (Figure $6 \mathrm{a}-$ $6 b)$. Once the two layers were interpolated onto a square grid (Figure 6a-b), line profiles were taken across the images. The line profiles were taken left to right along the $\mathrm{x}$ axis through the center of mass of the image in Figure $6 \mathrm{a}$ and b, with 100 line profiles spread along an area between $\pm 5 \AA$ from the center of mass. The images were then rotated by increments of 2 degrees around the center of mass of all the image was then rotated and the line profiles again extracted until the average wavelength extracted from the top and bottom layer matched. Using this method the wavelength and amplitude for the layers where found to be $\lambda_{1}=$ $14.64 \pm 0.61 \AA, A_{1}=0.73 \pm 0.08 \AA$, and $\lambda_{2}=14.64 \pm 0.54 \AA, A_{2}=0.77 \pm 0.06 \AA$, giving an average wavelength and amplitude of $\lambda=14.64 \pm 0.57 \AA$ and $A=0.75 \pm 0.07 \AA$.

To estimate the amount of strain, the primary-to-primary node distance and the secondary-to-secondary node distance in the patch were compared to the ideal distance based on the average angle of relative rotation in the whole image. The patch was chosen so that the primary-to-primary node distance and the secondary-to-secondary node distance are at approximately $90^{\circ}$ to each other in the patch, allowing the strain in each direction to be directly related to the compressive and tensile strain:

$$
\begin{gathered}
\varepsilon=\frac{\Delta L}{L} \\
v=\left|\frac{\varepsilon_{y}}{\varepsilon_{x}}\right|
\end{gathered}
$$



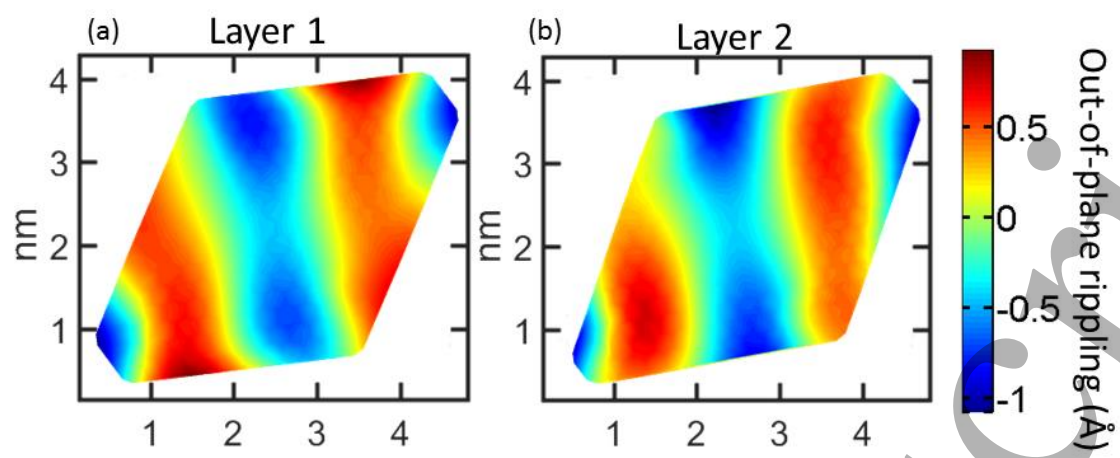

(c)
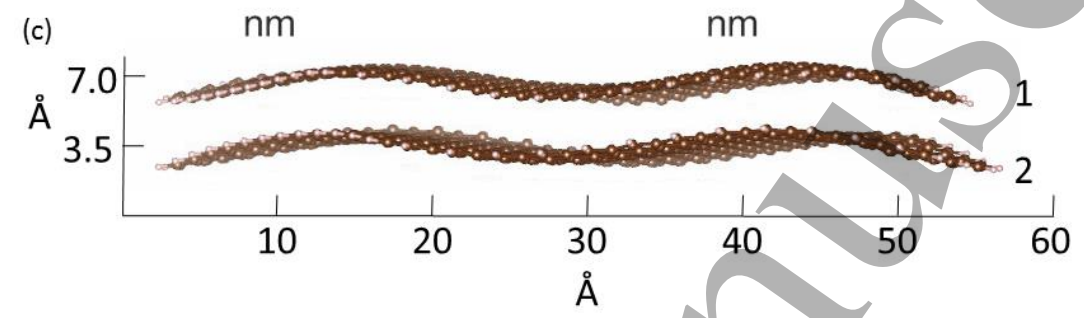

Figure 6. Displacement of the atoms perpendicular to the imaging-plane from the optimized positions produced by DFT for (a) the bottom layer, and (b) the top layer. (c) Atomistic model of the rippling relative to the layer separation.

In Eq. 5 [37], $\mathrm{L}$ is the predicted node-to-node distance based on the average angle of relative rotation $\left(4.45^{\circ}\right)$ and $\Delta \mathrm{L}$ is the difference between $\mathrm{L}$ and the measured node-to-node distance. Using Eq. 5, a tensile strain of $\varepsilon_{x}=6.78 \%$ and a compressiye strain of $\varepsilon_{y}=1.23 \%$ were calculated. Then, using Eq. 6 , an estimate of the in-plane Poisson's ratio $(v)$ can be determined as 0.18 for the patch. Using well-converged DFT calculations, we find that for both AA- and AB-stacked bilayers, as well as monolayer graphene, the in-plane Poisson ratio $(v)$ is 0.19 , in agreement with experimental measurements on graphene and graphite [38], indicating that there is practically no sensitivity to the number of layers for the in-plane Poisson's ratio in both theory and experiment.

Given the presence of strain-induced rippling in our sample, the question arises whether the observed rippling obeys continuum mechanics. For example, monolayer graphene appears to satisfy [4,5,39] or violate continuum mechanics [40,41], depending on the particular geometric setup conditions [42]. The continuum mechanics model of strained elastic sheets can be written either in terms of an applied tensile strain or in terms of a compressive strain. When uniaxial tensile strain is applied to an elastic sheet of length $L$ and thickness $t$, the wavelength $(\lambda)$ and amplitude $(A)$ of rippling are given by [43]:

$$
\begin{gathered}
\lambda=(2 \pi L t)^{1 / 2}\left[3\left(1-v^{2}\right) \varepsilon_{x}\right]^{-1 / 4} \\
A=(v L t)^{1 / 2}\left[16 \varepsilon_{x} /\left(3 \pi^{2}\left(1-v^{2}\right)\right)\right]^{1 / 4}
\end{gathered}
$$

where $v$ is the in-plane Poisson's ratio and $\varepsilon$ is the applied strain along the length. Likewise, for compressive strain of magnitude $\varepsilon_{y}$, the relevant equations are [39]:

$$
\begin{gathered}
\lambda=(2 \pi L t)^{1 / 2} v^{1 / 4}\left[3\left(1-v^{2}\right) \varepsilon_{y}\right]^{-1 / 4} \\
A=(v L t)^{1 / 2}\left[16 \varepsilon_{y} /\left(3 \pi^{2}\left(1-v^{2}\right)\right)\right]^{1 / 4} .
\end{gathered}
$$


(a)
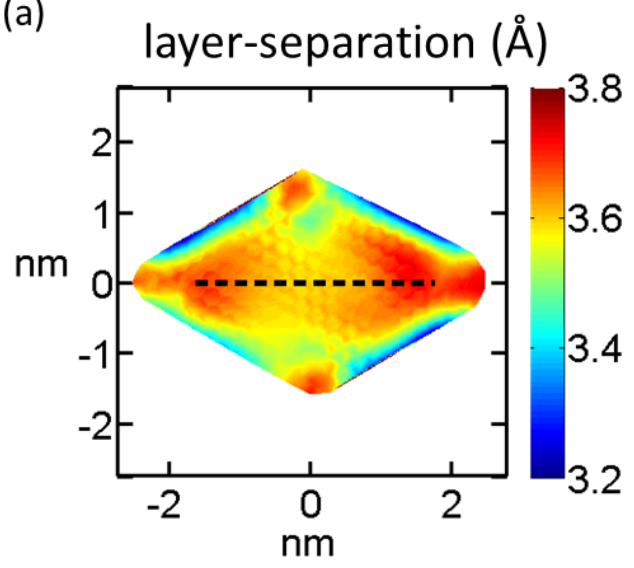

(b)

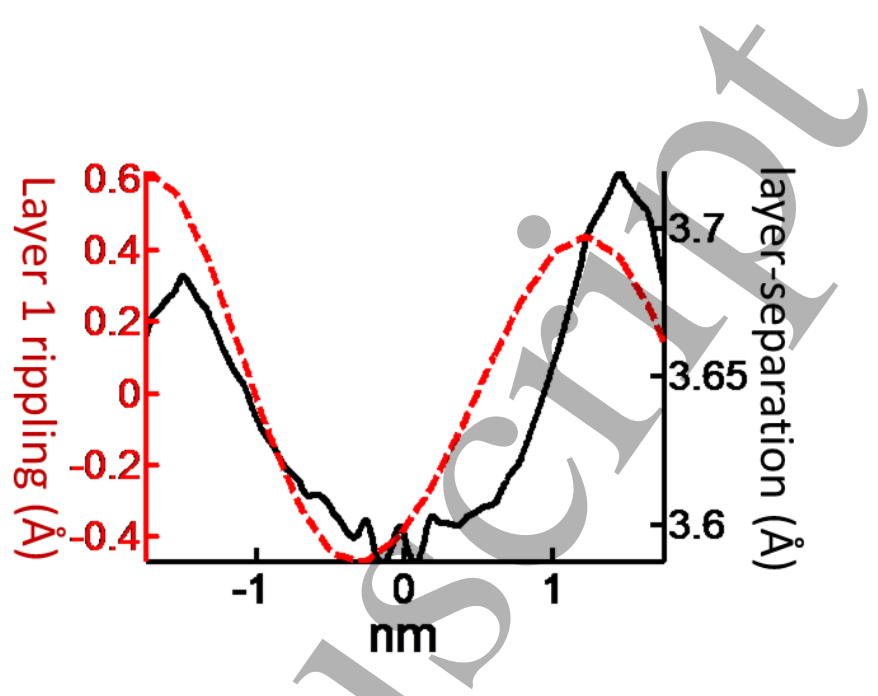

Figure 7. (a) Layer-separation between the two layers from the optimized positions. (b) Layerseparation (black solid line) and $z$ rippling for the top layer (red dotted line) across the line profile shown in (a).

Equations 7, 8 and 9, 10 are fundamentally related through the in-plane Poisson's ratio for the magnitude of tensile and compressive strains $\left(\varepsilon_{y}=v \varepsilon_{x}\right)$. In the current experimental setup, it is difficult to concretely define $L$. Furthermore, there is an ambiguity in how to define the thickness of atomically thin materials. Therefore, we can use Eqs. 7 and 8 to derive a relationship between $\lambda$ and $A$ in terms of $\varepsilon_{x}$ :

$$
A=\frac{\lambda}{\pi}\left(2 v \varepsilon_{x}\right)^{1 / 2}
$$

or Eqs. 9 and 10 to derive such a relationship in terms of $\varepsilon_{y}$ :

$$
A=\frac{\lambda}{\pi}\left(2 \varepsilon_{y}\right)^{1 / 2}
$$

Using the average extracted wavelength of $\lambda=14.64 \pm 0.57 \AA$ with a Poisson's ratio of 0.19 in Eq. 12 gives an expected amplitude of $A=0.73 \pm 0.03 \AA$. This expected amplitude and our extracted amplitude of $A=0.75 \pm 0.07 \AA$ agree within uncertainty, implying that the observed rippling in bilayer graphene is consistent with the continum theory of elasticity. Previous works have sparked the controversy as to whether rippling in graphene does obey [44] or does not obey [40] the continuum theory model; while in the present case for bilayer graphene, the rippling does obey the continuum theory.

Undulations in the $z$ direction in bilayer graphene with a low angle of relative rotation result in a change in the separation between the layers that coincides with the intensity change of the moire pattern [45]. To study the $z$ undulations caused by the angle of relative rotation between the two layers of bilayer graphene, the distance between the layers (Figure 6a-b) is used (Figure 7a). To measure these undulations in the current data, a series of line profiles were taken along a path between the centers of the primary moiré nodes in the patch. These line profiles show undulations with a wavelength that matches the primary-to-primary node distance and an amplitude of $0.07 \AA ̊$ (figure $7 \mathrm{~b}$ ). Semiclassical theory [45] predicts these undulations to have an amplitude of $\sim 0.1 \AA$, which is slightly larger than what is observed in our optimized unit cell. An interesting note is that the strain-based rippling along the same line profiles (Figure $7 \mathrm{~b}$ ) appears to have the same wavelength and orientation as the moiré rippling; however, the strain rippling amplitude is an order of magnitude larger. Due to the moiré undulations and the strain rippling being aligned, the moiré-induced undulations would be undetectable without the aid of DFT. 
This orientation match between the moiré-induced undulations and strain-based rippling might simply be a coincidence or an actual correlation.

\section{Outlook}

We have introduced a reconstructive analysis methodology to deduce the coordinates in all three spatial dimensions of the atoms that cannot resolved from one another in an experimental STEM image. The approach is not only able to deduce the $x y$ information that is lost in the STEM image, butalso provides information in the $z$ direction for which STEM provides little information. The $z$ information clearly shows rippling in the direction of strain as would be expected and in line with the continuum model. The method relies on the assumption of a defect-free lattice, but, using local-area correlation, it is possible to identify the location of potential defect areas. By inserting defects into the lattice computation and then reoptimizing the lattice, it is possible to identify the defect by optimizing the local correlation map in a selfconsistent manner.

While we have demonstrated success using a STEM image, the same approach can be performed using other atomic-resolution imaging techniques. Scanning tunneling microscopy (STM) could be an ideal candidate. STM can resolve individual atoms, but, like STEM, has a "blind spot" preventing full threedimensional measurement of positions in bilayer or multi-layer systems. STM has very high resolution in the $z$-direction normal to the surface and good resolution in the $x y$ plane of the surface, but is predominantly sensitive only to the outermost layer of atoms. STM provides little information about the lower layer in a bi-layer system. A number of atomically-resolved STM studies have identified rippling in both mono- and multi-layer graphene [46-48]. The details of the subsurface structure influences the surface layer at a level that can be observed by STM, i.e. changes in the vertical positions of atoms located above or between atoms below. The present reconstructive-analysis approach, combining data analysis of measured atomic positions with DFT modeling should identify the missing subsurface atomic structures and could resolve outstanding questions on friction and the origins of rippling.

\section{Conclusions}

We have demonstrated an approach for the recovery of atomic positions in three dimensions that cannot be determined from the raw data alone. Using a STEM image of bilayer graphene as the test case, the $x y$ positions of all atoms were recovered and the $z$ positions deduced. The $z$ positions were validated through the 2D cross-correlation of the simulated STEM image with a filtered raw-data image. Defects in the bilayer graphene sample were detected by the presence of areas of low correlation in the 2D cross-correlation map. It is then possible to use the correlation maps to identify the type of defects in a self-constant manner. In the present case, a defect was found and identified as a substitutional nitrogen. The $z$ positions show the presence of nanometer-scale rippling consistent with the continuum elastic model. Furthermore, we find that the angle of relative rotation in the bilayer causes additional moiré-induced undulations in the atomic positions. It should be possible to further extend this methodology to other types of atomically-resolved microscopy, such as STM.

\section{Acknowledgements}

The authors would like to thank Ivan Vlassiok for growing and providing the initial graphene sample and Jason Bonacum for help at the early stages of this project. A portion of this research was conducted at the Center for Nanophase Materials Sciences, which is a DOE Office of Science User Facility. This work was supported in part by Department of Energy grant DE-FG02-09ER46554, by National Science Foundation Grant DMR-1508433, and by the McMinn Endowment at Vanderbilt University. Supercomputer time was 
provided, in part, by the Extreme Science and Engineering Discovery Environment (XSEDE), which is supported by National Science Foundation Grant ACI-1053575. ARL and AYB were supported by the U.S. Department of Energy, Office of Science, Basic Energy Sciences, Materials Sciences and Engineering Division.

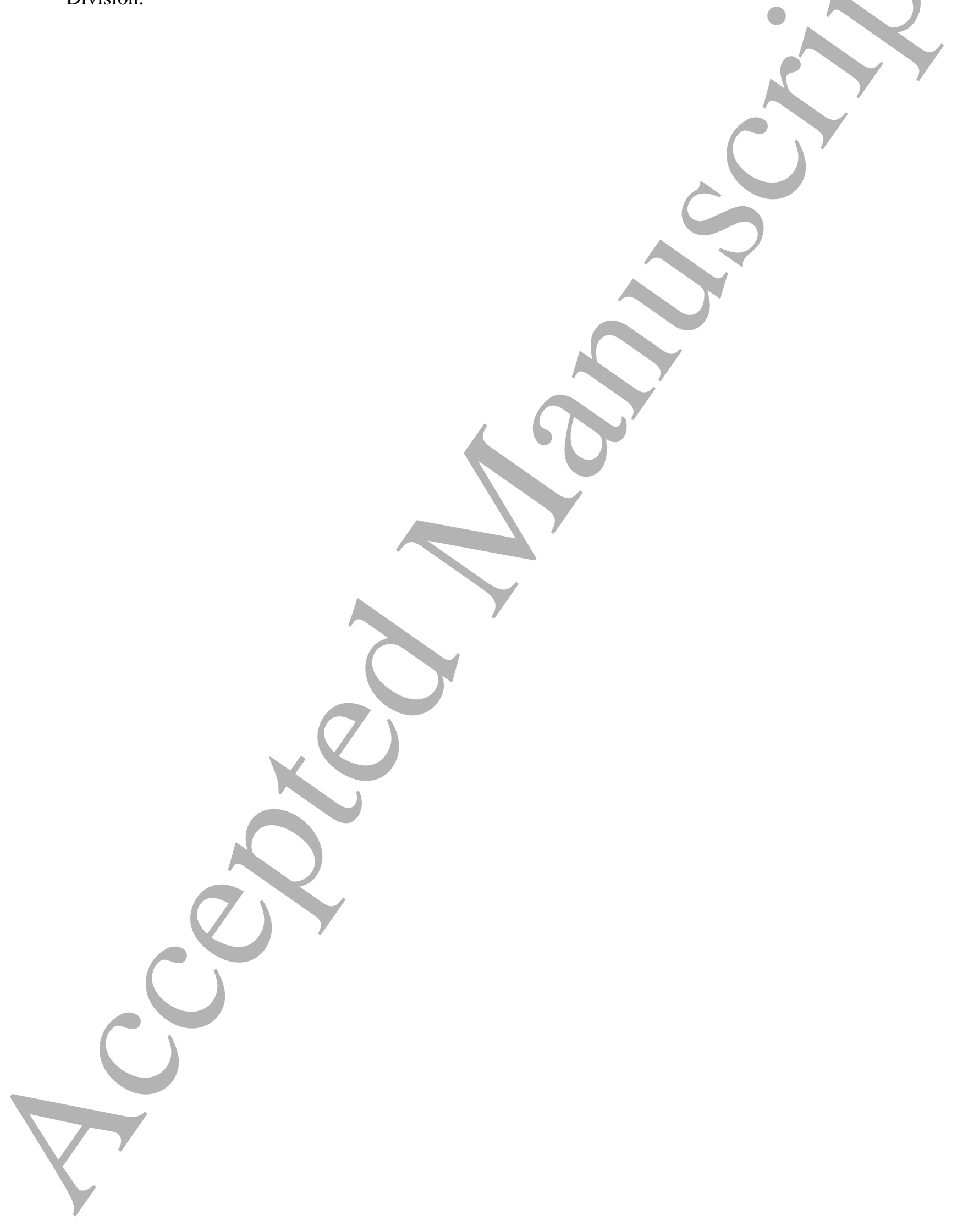




\section{References}

[1] Xu K, Cao P G and Heath J R 2009 Scanning Tunneling Microscopy Characterization of the Electrical Properties of Wrinkles in Exfoliated Graphene Monolayers Nano Lett.9 4446-51

[2] Levy N, Burke S A, Meaker K L, Panlasigui M, Zettl A, Guinea F, Neto A H C and Crommie M F 2010 Strain-Induced Pseudo-Magnetic Fields Greater Than 300 Tesla in Graphene Nanobubbles Science (80-. ). $329544-7$

[3] Nicholl R J T, Conley H J, Lavrik N V., Vlassiouk I, Puzyrev Y S, Sreenivas V P, Pantelides S T and Bolotin K I 2015 The effect of intrinsic crumpling on the mechanics of free-standing graphene Nat. Commun. 68789

[4] Bao W, Miao F, Chen Z, Zhang H, Jang W, Dames C and Lau C N 2009 Controlled ripple texturing of suspended graphene and ultrathin graphite membranes Nat. Nanotechnol. 4 562-6

[5] Meng L, Su Y, Geng D, Yu G, Liu Y, Dou R F, Nie J C and He L 2013 Hierarchy of graphene wrinkles induced by thermal strain engineering Appl. Phys. Lett. 103

[6] Yang L, Niu T, Zhang H, Xu W, Zou M, Xu L, Cao G and Cao A 2017 Self-assembly of suspended graphene wrinkles with high pre-tension and elastic property $2 D$ Mater. 4

[7] Xu S Y, Alidoust N, Belopolski I, Richardella A, Liu C, Neupane M, Bian G, Huang S H, Sankar R, Fang C, Dellabetta B, Dai W, Li Q, Gilbert M J, Chou F, Samarth N, Hasan M Z, Aikebaier F, Pertsova A, Canali C M, Charlebois M, Sénéchal D, Gagnon A M, Tremblay A M S, Wehling T O, Lichtenstein A I, Katsnelson M I, Overhauser A W, Yang H, Yang S H, Takahashi S, Maekawa S, Parkin S S P, Quay C H L, Weideneder M, Chiffaudel Y, Strunk C, Aprili M, Bistritzer R, MacDonald A H, Yamashita T, Takahashi S, Imamura H, Maekawa S, Kulik I 0, Cao Y, Fatemi V, Fang S, Watanabe K, Taniguchi T, Kaxiras E, Jarillo-Herrero P, Aslamazov L G, Larkin A I, Ovchinnikov Y N, ANDERSON P W, Bai C, Yang Y, Wehling T O, ŞaşIoğlu E, Friedrich C, Lichtenstein A I, Katsnelson M I, Blügel S, Schüler M, Rösner M, Wehling T O, Lichtenstein A I, Katsnelson M I and Yafet Y 2015 Unconventional superconductivity in magic-angle graphene superlattices Phys. Rev. B - Condens, Matter Mater. Phys. 108 1-10

[8] Cao Y, Fatemi V, Demir A, Fang S, Tomarken S L, Luo J Y, Sanchez-Yamagishi J D, Watanabe K, Taniguchi T, Kaxiras E, Ashoori R C and Jarillo-Herrero P 2018 Correlated insulator behaviour at half-filling in magic-angle graphene superlattices Nature 556 80-4

[9] Lin J, Fang W, Zhou W, Lupini A R, Idrobo J C, Kong J, Pennycook S J and Pantelides S T 2013 AC/AB stacking boundaries in bilayer graphene Nano Lett. 13 3262-8

[10] Castro E V., Novoselov K S, Morozov S V., Peres N M R, dos Santos J M B L, Nilsson J, Guinea F, Geim A K and Neto A H C 2007 Biased Bilayer Graphene: Semiconductor with a Gap Tunable by the Electric Field Effect Phys. Rev. Lett. 99216802

[11] O'Hara A, Kahn R E, Zhang Y-Y and Pantelides S T 2017 Defect-mediated leakage in lithium intercalated bilayer graphene AIP Adv. 745205

[12] Zhang Y, Tang T-T, Girit C, Hao Z, Martin M C, Zettl A, Crommie M F, Shen Y R and Wang F 2009 Direct observation of a widely tunable bandgap in bilayer graphene Nature 459 820-3

[13] Regan W, Alem N, Alemán B, Geng B, Girit Ç, Maserati L, Wang F, Crommie M and Zettl A 2010 A direct transfer of layer-area graphene Appl. Phys. Lett. 96113102 
[14] Mao Y and Zhong J 2008 Structural, electronic and magnetic properties of manganese doping in the upper layer of bilayer graphene. Nanotechnology 19205708

[15] Borisevich A Y, Chang H J, Huijben M, Oxley M P, Okamoto S, Niranjan M K, Burton J D, Tsymbal E Y, Chu Y H, Yu P, Ramesh R, Kalinin S V. and Pennycook S J 2010 Suppression of Octahedral Tilts and Associated Changes in Electronic Properties at Epitaxial Oxide Heterostructure Interfaces Phys. Rev. Lett. 10587204

[16] Ishikawa R, Lupini A R, Hinuma Y and Pennycook S J 2015 Large-angle illumination STEM: Toward three-dimensional atom-by-atom imaging Ultramicroscopy 151 122-9

[17] Krivanek O L, Chisholm M F, Nicolosi V, Pennycook T J, Corbin G J, Dellby N, Murfitt M F, Own C S, Szilagyi Z S, Oxley M P, Pantelides S T and Pennycook S J 2010 Atom-by-atom structural and chemical analysis by annular dark-field electron microscopy Nature 464 571-4

[18] Shen X, Hernández-Pagan E A, Zhou W, Puzyrev Y S, Idrobo J-C, Macdonald J E, Pennycook S J and Pantelides S T 2014 Interlaced crystals having a perfect Bravais lattice and complex chemical order revealed by real-space crystallography Nat. Commun. 55431

[19] Zhou W, Lee J, Nanda J, Pantelides S T, Pennycook S J and Idrobo J-C 2012 Atomically localized plasmon enhancement in monolayer graphene Nat. Nanotechnol. 7 161-5

[20] Wijk M M van, Schuring A, Katsnelson M I andFasolino A 2015 Relaxation of moiré patterns for slightly misaligned identical lattices: graphene on graphite 2D Mater. 234010

[21] Vlassiouk I, Fulvio P, Meyer H, Lavrik N, Dai S, Datskos P and Smirnov S 2013 Large scale atmospheric pressure chemical vapor deposition of graphene Carbon N. Y. 54 58-67

[22] Anon 2um holes with 2um spacing (657-200-AU QUANTIFOIL SUBSTRATE, 200M)No Title

[23] Krivanek O L, Corbin G J, Dellby N, Elston B F, Keyse R J, Murfitt M F, Own C S, Szilagyi Z S and Woodruff J W 2008 An electron microscope for the aberration-corrected era Ultramicroscopy 108 179-95

[24] Witkin A 1984 Scale-space filtering: A new approach to multi-scale description ICASSP ' 84. IEEE Int. Conf. Acoust. Speech, Signal Process. 9 150-3

[25] Ester M, Kriegel H P, Sander J and Xu X 1996 A Density-Based Algorithm for Discovering Clusters in Large Spatial Databases with Noise Proc. 2nd Int. Conf. Knowl. Discov. Data Min. 226-31

[26] Nishiyima Y and Oster G 1964 Moiré Patterns: Their Application to Refractive Index and Refractive Index Gradient Measurements J. Opt. Soc. Am. 54 1-5

[27] Somnath S, Smith C R, Kalinin S V., Chi M, Borisevich A, Cross N, Duscher G and Jesse S 2018 Feature extraction via similarity search: application to atom finding and denoising in electron and scanning probe microscopy imaging Adv. Struct. Chem. Imaging 43

[28] McNemar Q 1947 Note on the sampling error of the difference between correlated proportions or percentages Psychometrika 12 153-7

[29] Kresse G and Furthmüller J 1996 Efficient iterative schemes for ab initio total-energy calculations using a plane-wave basis set Phys. Rev. B 54 11169-86

[30] Perdew J P, Burke K and Ernzerhof M 1997 Erratum: Generalized gradient approximation made simple (Physical Review Letters (1996) 77 (3865)) Phys. Rev. Lett. 781396 
[31] Grimme S, Antony J, Ehrlich S and Krieg H 2010 A consistent and accurate ab initio parametrization of density functional dispersion correction (DFT-D) for the 94 elements $\mathrm{H}-\mathrm{Pu} \mathrm{J}$. Chem. Phys. 132154104

[32] Becke A D and Johnson E R 2005 A density-functional model of the dispersion interaction J. Chem. Phys. 123154101

[33] Lebedeva I V., Lebedev A V., Popov A M and Knizhnik A A 2017 Comparison of performance of van der Waals-corrected exchange-correlation functionals for interlayer interaction in graphene and hexagonal boron nitride Comput. Mater. Sci. 128 45-58

[34] Blöchl G, Joubert D, Savrasov S Y, Humphreys C J, Sutton A P, Monconduit L, Mahdoud A, Dominko R, Tendeloo G Van, Hermann R P, Tarascon J-M, Komaba S, Gonbeau D, Novak P, Tendeloo G Van, Hermann R P and Tarascon J-M 1994 Projector augmented-wave method. Phys. Rev. B. Condens. Matter 50 17953-79

[35] Kresse G and Joubert D 1999 From ultrasoft pseudopotentials to the projector augmented-wave method Phys. Rev. B 59 1758-75

[36] Koch C 2002 Determination of core structure periodicity and point defect density along dislocations (Arizona State University)

[37] Fung Y C 1965 Foundation of Solid Mechanics

[38] Politano A and Chiarello G 2015 Probing the Young's modulus and Poisson's ratio in graphene/metal interfaces and graphite: a comparative study Nano Res. 8 1847-56

[39] Wang Z and Devel M 2011 Periodic ripples in suspended graphene Phys. Rev. B 83125422

[40] Tapasztó L, Dumitrică T, Kim S J, Nemes-Incze P, Hwang C and Biró L P 2012 Breakdown of continuum mechanics for nanometre-wavelength rippling of graphene Nat. Phys. 8 739-42

[41] Bai K-K, Zhou Y, Zheng H, Meng L, Peng H, Liu Z, Nie J-C and He L 2014 Creating OneDimensional Nanoscale Periodic Ripples in a Continuous Mosaic Graphene Monolayer Phys. Rev. Lett. 11386102

[42] Liu F and Wang T C 2016 Quantized phenomena in graphene nanoripples Carbon N. Y. 961175 80

[43] Cerda E, Mahadevan L, Moon M-W, Oh K H, Kim K-S, Allen H G, Biot M A, Biot M A, Bowden N, Brittain S, Evans A G, Hutchinson J W, Whitesides G M, Cerda E, Mahadevan L, Chan E P, Smith E J, Hayward R C, Crosby A J, Chen X, Hutchinson J W, Efimenko K, Genzer J, Groenewold J, Gough G S, Elam C F, Bruyne N A de, Hoff N J, Mautner S E, Huang R, Huang Z Y, Hong W, Suo Z, Huck W T S, Kim K-S, Kim J, Kim S O, Earmme Y Y, Kim K-S, Lee D, Triantafyllidis N, Barber J R, Thouless M D, Moon M-W, Ogden R W, Ogden R W, Rahmawan Y, Moon M-W, Kim K-S, Lee K R, Suh K Y, Rivlin R S, Röll K, Shield T W, Kim K-S, Shield R T, Song J, Stafford C M, Steigmann D J, Ogden R W, Timoshenko S P, Gere J M, Yoo S H, Cohen C and Hui C-Y 2003 Geometry and physics of wrinkling. Phys. Rev. Lett. 9074302

[44] Wei X, Fragneaud B, Marianetti C A and Kysar J W 2009 Nonlinear elastic behavior of graphene: Ab initio calculations to continuum description Phys. Rev. B 80205407

[45] Bistritzer R and MacDonald A H 2011 Moire bands in twisted double-layer graphene Proc. Natl. Acad. Sci. 108 12233-7

[46] Choi J S, Kim J-S, Byun I-S, Lee D H, Lee M J, Park B H, Lee C, Yoon D, Cheong H, Lee K H, 
Son Y-W, Park J Y and Salmeron M 2011 Friction Anisotropy-Driven Domain Imaging on Exfoliated Monolayer Graphene Science (80-. ). 333 607-10

[47] Gallagher P, Lee M, Amet F, Maksymovych P, Wang J, Wang S, Lu X, Zhang G, Watanabe K, Taniguchi T and Goldhaber-Gordon D 2016 Switchable friction enabled by nanoscale selfassembly on graphene Nat. Commun. 710745

[48] Zan R, Bangert U, Muryn C, Mattocks P, Hamilton B and Novoselov K S 2012 Scanning Tunnelling Microscopy of Suspended Graphene J. Phys. Conf. Ser. 37112070 